\title{
Endodontic Management of Tooth with Open Apex Using MTA as an Apical Barrier: A Case Report
}

\author{
Rakhi P Veedu ${ }^{1}$, Radhakrishnan Nair K², Praveena Geetha ${ }^{3}$, Merin Joseph ${ }^{4}$, Emi George ${ }^{5}$
}

\begin{abstract}
Background: Endodontic therapy of a tooth with open apex and necrotic pulp presents multiple challenges during treatment. Microbial elimination from chronic lesions makes treatment all the more difficult. Apexification and pulp revascularization are the treatment options available. Apexification with calcium hydroxide has certain disadvantages and mineral trioxide aggregate (MTA) may be considered as a promising alternative. Case description: This case report describes the management of a symptomatic maxillary left central incisor with immature apex and a chronic periapical lesion. Sodium hypochlorite of $3 \%$ and EDTA were used as irrigants for microbial elimination. Repeated intracanal dressings with calcium hydroxide were given for better periapical healing. MTA was used for apical barrier formation. After confirming the hard set of MTA, obturation was done with thermoplasticized gutta-percha and access cavity sealed with composite resin restoration.

Conclusion: In this case, MTA helped to form an apical barrier in the immature necrotic maxillary central incisor and showed a good clinical and radiographic success. The use of irrigants and calcium hydroxide as an intracanal medicament promoted the periapical healing of the chronic apical periodontitis.
\end{abstract}

Keywords: Apexification, Calcium hydroxide, Immature teeth, MTA, Open apex.

Conservative Dentistry and Endodontic Journal (2019): 10.5005/jp-journals-10048-0042

\section{BACKGROUND}

Endodontic therapy of a tooth with open apex and necrotic pulp presents multiple challenges during treatment. Cleaning and disinfection of the infected root canal cannot be done with standard root canal protocols. A lower strength of irrigants is used because of the danger of its extrusion through the apex of the immature teeth. Obturation of the root canal system is difficult as there is no apical barrier for containing the obturating material without impinging on the periodontal tissues. Hence, obtaining a fluid-tight seal is often difficult. Also, the presence of thin roots increases the susceptibility to fracture.

Pulp revascularization in necrotic immature teeth is possible provided proper disinfection of root canal space is achieved and the conditions are favorable. It results in increased dentin wall width and root end development. However, the treatment can take many months to years with multiple clinical appointments and patient compliance can be a problem.

Apexification with calcium hydroxide is the most common treatment option for necrotic teeth with immature roots. However, calcium hydroxide shows certain limitations like incomplete calcification of dentinal bridge and prolonged treatment duration which may present problems with patient compliance. ${ }^{1}$ There are chances for reinfection due to loss of coronal seal in between appointments and also the long-term use of calcium hydroxide predisposes the tooth to fracture as it decreases the fracture resistance of the tooth. ${ }^{2-4}$

Mineral trioxide aggregate is a promising alternative material for the apexification procedure and has been shown to be a very effective root-filling material for sealing immature root canals with open apices. ${ }^{5}$ It is also used as a root end-filling material and perforation repair material due to its good canal sealing property and biocompatibility. It facilitates the formation of dentinal bridges and cementum, and regeneration of the periodontal ligament. It has the ability to stimulate cytokine release from the bone cells,
${ }^{1-5}$ Department of Conservative Dentistry and Endodontics, Azeezia College of Dental Sciences and Research, Kollam, Kerala, India

Corresponding Author: Rakhi P Veedu, Department of Conservative Dentistry and Endodontics, Azeezia College of Dental Sciences and Research, Kollam, Kerala, India, Phone: +91 8495013778, e-mail: rakhisanthu@gmail.com

How to cite this article: Veedu RP, Nair KR, et al. Endodontic Management of Tooth with Open Apex Using MTA as an Apical Barrier: A Case Report. Cons Dent Endod J 2019;4(1):14-17.

Source of support: Nil

Conflict of interest: None

indicating that it actively promotes hard tissue formation and facilitates periradicular healing. ${ }^{6}$

Microbial elimination in chronic periapical lesions is another challenge for clinicians. However, adequate disinfection of the root canal system with mechanical debridement in combination with intracanal medicaments helps in nonsurgical treatment of such lesions and favors regression and healing. ${ }^{7}$ Calcium hydroxide has antimicrobial property and has been widely used as an interappointment intracanal medicament. ${ }^{8}$ Calcium hydroxide when given as an interim dressing in the presence of large and chronic periapical lesions can create an environment more favorable to healing and encourage osseous repair. ${ }^{9}$

This case report describes the management of a symptomatic tooth with immature apex and a chronic periapical lesion with calcium hydroxide used as an intracanal medicament and MTA used for apical barrier formation.

\section{Case Description}

A 23-year-old male reported at the Department of Conservative Dentistry and Endodontics, with the complaint of intermittent pain

() The Author(s). 2019 Open Access This article is distributed under the terms of the Creative Commons Attribution 4.0 International License (https://creativecommons. org/licenses/by-nc/4.0/), which permits unrestricted use, distribution, and non-commercial reproduction in any medium, provided you give appropriate credit to the original author(s) and the source, provide a link to the Creative Commons license, and indicate if changes were made. The Creative Commons Public Domain Dedication waiver (http://creativecommons.org/publicdomain/zero/1.0/) applies to the data made available in this article, unless otherwise stated. 
on the upper front tooth. Pain aggravated on biting. The patient gave a history of trauma 9-10 years back. Root canal treatment was initiated 2 years before. The patient underwent treatment for a few appointments but failed to report to the dentist for completion of treatment.

The patient had no relevant medical history. This was a case of Ellis class III fracture on the maxillary left central incisor with an access cavity preparation and there was a loss of coronal seal. On palpation, there was mild pain in the labial sulcus corresponding to the apical region of 21 . The tooth also showed mild tenderness to percussion. All teeth except 21 elicited a normal response on thermal as well as electric pulp testing. Periapical radiograph of 21 revealed an apex which is incompletely formed with a periapical radiolucency of approximately $4 \times 3 \mathrm{~mm}$. A faint radiopacity was seen at the apex suggestive of remnants of extruded intracanal medicament. It was diagnosed to be a case of chronic apical periodontitis in relation to the maxillary left central incisor with an open apex.

In the first appointment, after rubber dam isolation, the existing access cavity was refined with a safe-end tapered bur (Endo Z bur, Dentsply Maillefer) (Fig. 1). The working length was determined using a no. $60 \mathrm{~K}$ file (Mani Inc., Japan) (Fig. 2). A definite stop at the apex could not be achieved clinically. Biomechanical preparation of the root canal was done with minimal instrumentation using standard hand instruments up to a size of $80 \mathrm{~K}$ file (Mani Inc., Japan). The root canal was irrigated alternately with a $5.25 \%$ sodium hypochlorite solution (Associated Clinical Aids, India) and normal saline (Eurolife Healthcare Pvt Ltd, India).

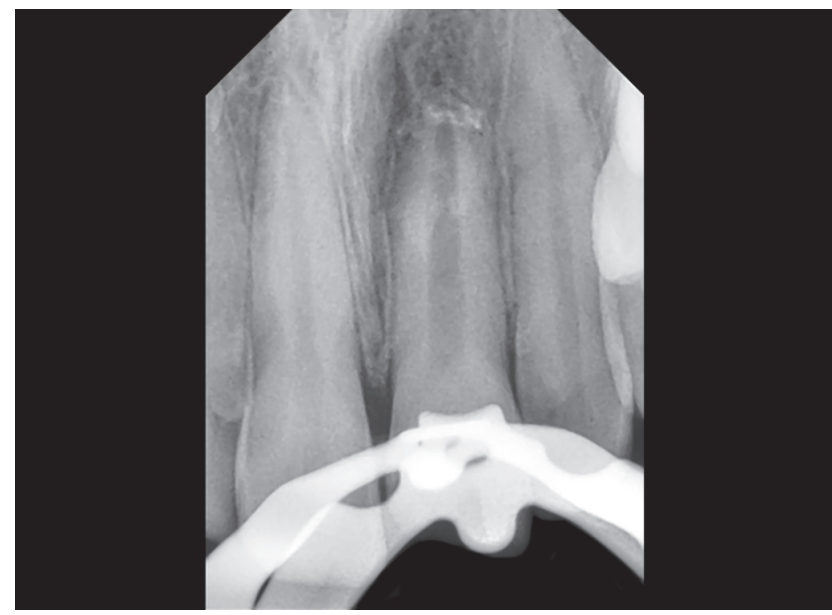

Fig. 1: Initial radiograph

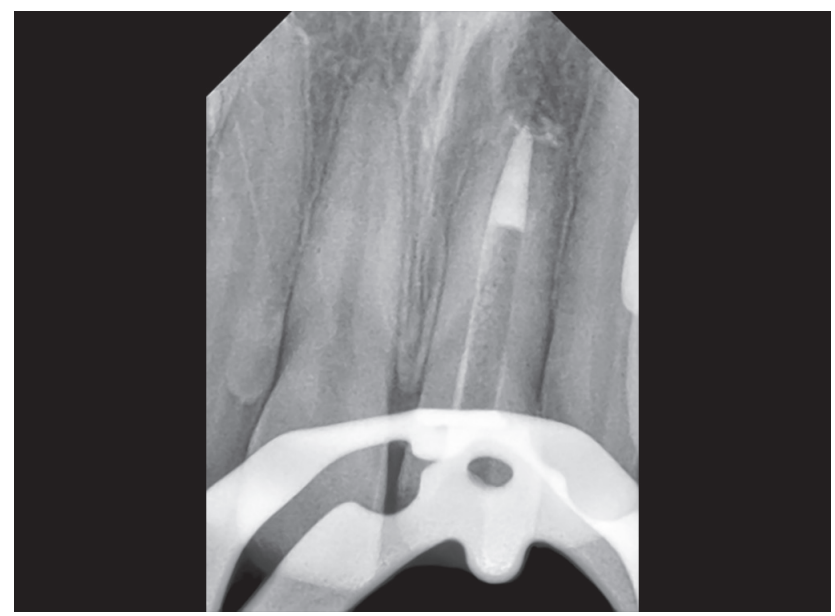

Fig. 3: Mineral trioxide aggregate plug confirmed
A final irrigation was done with 17\% EDTA (Anabond Stedman Pharma, India). Calcium hydroxide powder (Prevest Dent Pro, India) mixed with normal saline in a creamy mix was placed in the canal as an intracanal medicament and the patient was recalled periodically for evaluation. Two dressings of calcium hydroxide were repeated at 2 weeks intervals. The tooth was mildly tender to percussion during this period.

In the fourth appointment, the patient was completely asymptomatic. Calcium hydroxide was eliminated by mechanical instrumentation and rinsed out of root canals by means of saline irrigation. The canal was dried with absorbent paper points.

MTA (Dentsply Tulsa Dental specialties) was mixed according to the instructions from the manufacturer and carried to the canal with an amalgam carrier. Hand and finger pluggers (Mani Inc.) were used to condense the MTA. Too much apical pressure was avoided to prevent extrusion through apex. An apical plug of $4 \mathrm{~mm}$ of MTA was placed and confirmed radiographically (Fig. 3). A sterile cotton pellet moistened with saline was placed over the canal orifice and the access cavity was sealed with a temporary restoration. After 2 days, the cotton pellet was removed, the hard set of MTA was confirmed with a plugger, and the remainder of the root canal was obturated with the thermoplasticized gutta-percha (Denjoy obturation system) and $\mathrm{AH}$ plus sealer (Dentsply Maillefer) using vertical compaction (Fig. 4). A postendodontic restoration with composite resin (Tetric N Ceram, Ivoclar) was done. The patient was recalled periodically after

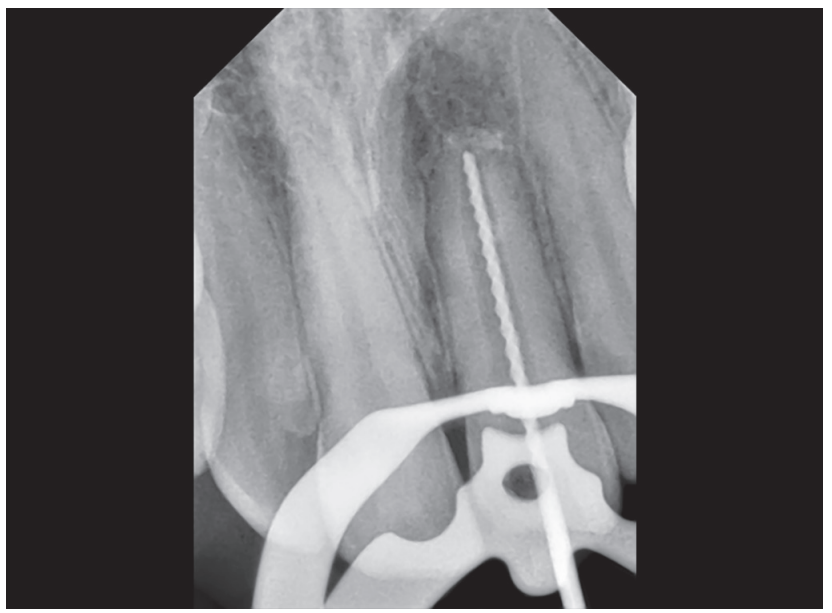

Fig. 2: Working length radiograph

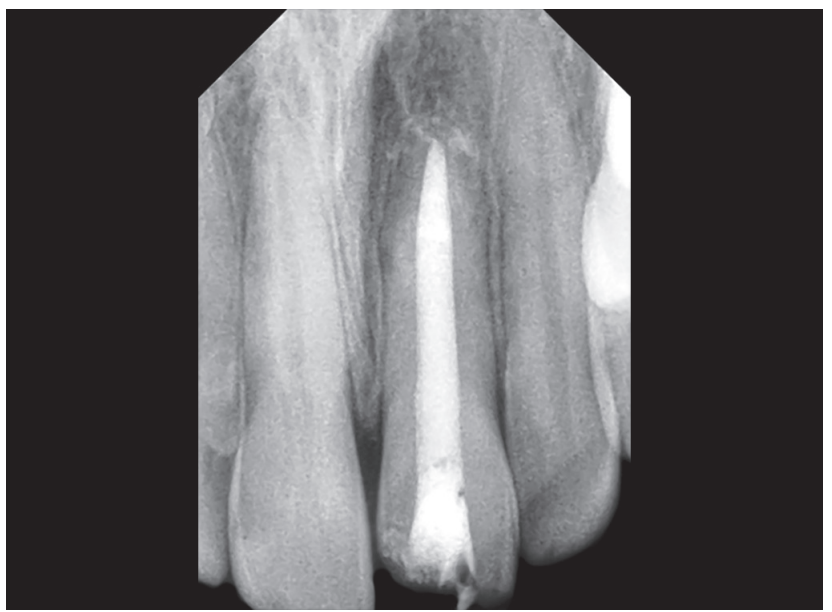

Fig. 4: Immediate postoperative radiograph 


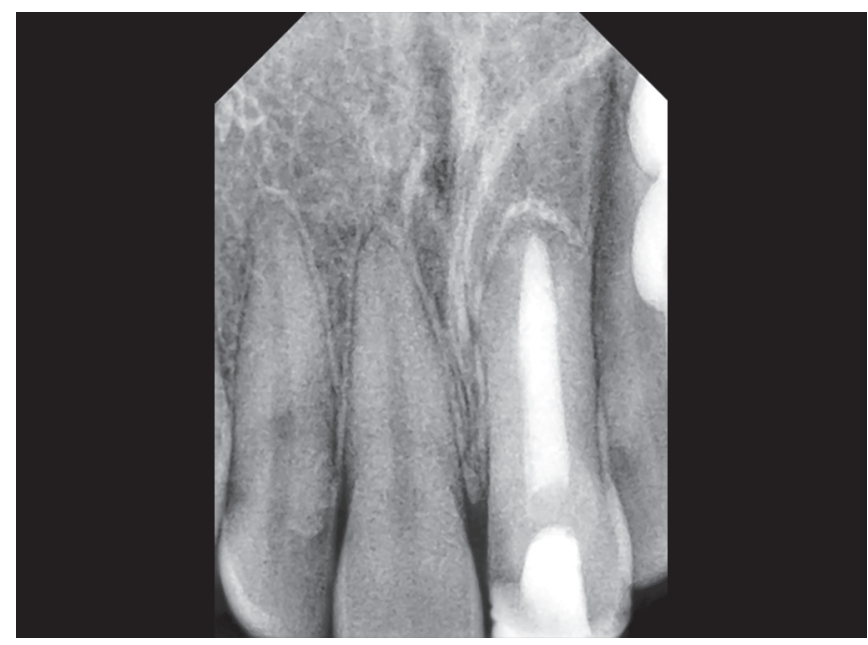

Fig. 5: Review after 18 months

1 month, 6 months, and 1 year to assess the periradicular healing clinically and radiographically. The patient was completely asymptomatic and the radiograph showed evidence of periapical healing (Fig. 5).

\section{Discussion}

Traumatic injuries to young permanent teeth result in pulpal inflammation or necrosis and subsequent incomplete development of dentinal wall and root apices. ${ }^{10}$ In such cases, maintaining the proper apical barrier with the three-dimensional seal becomes difficult. Management of such teeth becomes further complicated when there is an associated periapical infection.

Endodontic treatment options for such teeth consist of the conventional apexification procedure or revascularization. ${ }^{11}$ Pulp revascularization remains a good treatment option for such cases with the advantage of further root development and reinforcement of dentinal walls but it is generally limited to patients aged 8-16 years. ${ }^{12-14}$ Here, taking into consideration the age of the patient, the treatment option of apexification was decided. The aim of apexification is the production of mineralized apical barrier and to prevent the passage of toxins and bacteria into periapical tissues from the root canal. Also, this barrier is necessary to allow compaction of root-filling material.

Calcium hydroxide has been used traditionally for apexification. But it has certain drawbacks like the length of time needed to form apical barrier ranging from 5 to 20 months, patient compliance, chances for reinfection due to loss of temporary restoration in between appointments, and decreased fracture resistance of the tooth. ${ }^{15}$

The MTA apexification procedure was completed in two visits compared to the multiple visits' calcium hydroxide apexification. ${ }^{16}$ MTA is relatively noncytotoxic and stimulates cementogenesis. It creates a highly alkaline aqueous environment by leaching of calcium and hydroxyl ions. ${ }^{17}$ This property makes the MTA bioactive by forming hydroxyapatite in the presence of phosphate-containing fluids. ${ }^{18}$ MTA has the ability to induce the cementum-like hard tissue when used adjacent to the periradicular tissue. MTA is a promising material as a result of its superior sealing property, its ability to set in the presence of blood, and its biocompatibility. ${ }^{5}$ Moisture contamination at the apex of the tooth before barrier formation is often a problem with other materials used in apexification.
The presence of moisture does not affect the sealing ability of MTA since it is hydrophilic. Studies demonstrate that MTA produces a more predictable and consistent hard tissue formation compared to calcium hydroxide. ${ }^{19}$

The barrier produced by calcium hydroxide apexification has been reported to be incomplete having Swiss cheese appearance and can allow apical microleakage. Torneck et al. have indicated that when apical closure takes place clinically, with calcium hydroxide, there is no complete bridging of the apex histologically. Periapical inflammation persists about the apices of many teeth because the necrotic tissue exists in corners and crevices of the bridge. ${ }^{20}$

Other than the presence of an open apex another challenge, in this case, was to treat the chronic periapical lesion associated with it. Mechanical debridement alone may be unable to completely disinfect the root canal system. Bacteria which survive root canal instrumentation may proliferate between appointments and colonize the root canal. Therefore, calcium hydroxide was used as an intracanal medicament between appointments to eliminate microorganisms. Antimicrobial activity of calcium hydroxide is attributed to dissociation into calcium and hydroxyl ions in a high $\mathrm{pH}$ environment which inhibits enzymatic activities essential for bacterial survival, metabolism, growth, and cellular division. Repeated dressings of calcium hydroxide at 2-week intervals were given until the patient was asymptomatic.

In this case, obturation was done using thermoplasticized gutta-percha. It helps in achieving a better seal and also prevents compaction forces on the thin dentinal walls, unlike the lateral condensation technique.

The patient was reviewed at 3 months, 6 months, and 1 year. Clinical and radiographic outcomes were satisfactory.

\section{CONCLUSION}

In this case, MTA helped to form an apical barrier in the immature necrotic maxillary central incisor and showed a good clinical and radiographic success. The use of irrigants and calcium hydroxide as an intracanal medicament promoted the periapical healing of the chronic apical periodontitis.

\section{Clinical Significance}

Adequate infection control with mechanical debridement and irrigants in combination with intracanal medicaments helps in nonsurgical treatment of chronic lesions and favors regression and healing. Apical barrier with MTA helps to contain the obturating material without impinging on the periodontal tissues and obtaining a fluid-tight seal in immature teeth with an open apex.

\section{References}

1. Taneja S, Kumari M, et al. Management of open apex in anterior teeth using MTA-report of three cases. J Dent Spec 2013;1(1):114-119.

2. Magura ME, Kafrawy AH, et al. Human saliva coronal microleakage in obturated root canals: an in vitro study. J Endod 1991;17:324-331. DOI: 10.1016/S0099-2399(06)81700-0.

3. Andresen JO, Munksgaard EC, et al. Comparison of fracture resistance in root canals of immature sheep teeth after filling with calcium hydroxide or MTA. Dent Traumatol 2006;22:154-156. DOI: 10.1111/j.1600-9657.2006.00419.x.

4. Andreasen JO, Farik B, et al. Long term calcium hydroxide as a root canal may increase risk of root fracture. Dent Traumatol 2002;18:134-137. 
5. Muhammed AH, Azzaldeen A, et al. Mineral trioxide aggregate (MTA) in apexification. Endodontology 2013;25(2):97-101.

6. Koh ET, Pittford TR, et al. Mineral trioxide aggregate stimulates cytokine production in human osteoblasts. J Bone Min Res 1995;10S:S406.

7. Subramanyam $D$, Somasundaram S. Evaluation of the stability and antibacterial activity of various concentrations of triple antibiotic paste against streptococcus mutans-an in vitro study. Int J Curr Med Pharm Res 2017;3(5):1696-1699.

8. Dixit S, Dixit A, et al. Non surgical treatment of two periapical lesions with calcium hydroxide using two different vehicles. Case Rep Dent 2014;2014:901497. DOI: 10.1155/2014/901497.

9. Milosevic A. Calcium hydroxide in restorative dentistry. J Dent 1991;19(1):3-13.

10. Anantharaj $A$, Praveen $P$, et al. Challenges in pulpal treatment of young permanent teeth-a review. J Dent Sci Res 2011;2(1):142-155.

11. Wigler R, Kaufman A, etal. Revascularization: a treatment for permanent teeth with necrotic pulp and incomplete root development. J Endod 2013;39(3):319-326. DOI: 10.1016/j.joen.2012.11.014

12. Nygaard-Ostby B, Hjortdal O. Tissue formation in the root canal following pulp removal. Scand J Dent Res 1971;79:333-349.

13. Banchs F, Trope M. Revascularization of immature permanent teeth with apical periodontitis: new treatment protocol? J Endod 2004;30:196-200. DOI: 10.1097/00004770-200404000-00003.
14. Trope M. Treatment of the immature tooth with a non-vital pulp and apical periodontitis. Dent Clin North Am 2010;54:313-324. DOI: 10.1016/j.cden.2009.12.006.

15. Sheehy EC, Roberts GJ. Use of calcium hydroxide for apical barrier formationand healing in non-vital immature permanent teeth: a review. Br Dent J 1997;183(7):241-246. DOI: 10.1038/sj.bdj.4809477.

16. Gaitonde P, BishopK. Apexification with mineral trioxide aggregate:an overview of the material and technique. Eur J Prosthodont Restor Dent 2007;15(1):41-45.

17. Fridland $M$, Rosado R. Mineral trioxide aggregate (MTA) solubility and porosity with different water-to-powder ratios. J Endod 2003;29: 814-817. DOI: 10.1097/00004770-200312000-00007.

18. Sarkar NK, Caicedo R, et al. Physicochemical basis of the biologic properties of mineral trioxide aggregate. J Endod 2005;31:97-100.

19. Shabahang $S$, Torabinejad $M$, et al. A comparative study of root-end induction using osteogenic protein-1, calcium hydroxide, and mineral trioxide aggregate in dogs. J Endod 1999;25:1-5. DOI: 10.1016/S00992399(99)80388-4.

20. Torneck CD, Smith JS, et al. Biologic effects of endodontic procedures on developing incisor teeth. IV. Effect of debridement procedures and calcium hydroxide-camphorated parachlorophenol paste in the treatment of experimentally induced pulp and periapical disease. Oral Surg Oral Med Oral Pathol 1973;35(4):541-554. DOI: 10.1016/0030-4220(73)90012-1. 